\title{
钯催化卡宾桥联碳氢键活化构筑二苯并 $\mathcal{E}$-内酰胺
}

\author{
阎旭飞夏莹*
}

(四川大学华西公共卫生学院/华西第四医院 生物治疗国家重点实验室 成都 610041)

\section{A Modular Approach to Dibenzo-Fused $\mathcal{E}$-Lactams: Palladium Carbene Bridging $\mathrm{C}-\mathrm{H}$ Activation}

\author{
Yan, Xufei Xia, Ying* \\ (West China School of Public Health and West China Fourth Hospital, and State Key Laboratory of Biotherapy, \\ Sichuan University, Chengdu 610041)
}

含有二苯并七元含氮杂环结构的三环类化合物具 有潜在的生物活性, 在药物合成中具有重要的应用价 值 ${ }^{[1]}$. 二苯并 $[b, e]$ 氮杂草-6-酮作为其中一类骨架分子, 传统合成方法大多局限于预先活化底物的分子内偶联 关环，如含胺基和羧基的二芳基甲烷的内酰胺化反应 ${ }^{[2]}$; 醋酸钯催化的含酰胺基和卤素的二芳基甲烷的 $N$-芳基 化反应 ${ }^{[3]}$; 三价碘试剂催化的苯甲酰胺类化合物 $\mathrm{C}\left(\mathrm{sp}^{2}\right)$ 一 $\mathrm{H}$ 氨基化反应 ${ }^{[4]}$; 氯化亚铁催化的葱酮和叠氮 三甲基硅烷的扩环内酰胺化反应 ${ }^{[5]}$. 另外, 苯炔和 2-吲 哚酮经过选择性插入扩环反应也能合成此类化合物, 但 是反应只有中等收率且苯炔的底物类型很受限制 ${ }^{[6]}$ (Scheme 1). 总的来说, 二苯并 $[b, e]$ 氮杂草-6-酮骨架分 子的合成方法还是相对受限, 不利于此类化合物后续的 药物活性筛选, 发展简单高效的合成方法仍然需要进一 步探索.

近年来, 钯催化卡宾参与的偶联反应获得了极大的 发展，相比于重氮化合物的不稳定性和一定的操作危险 性, $N$-对甲苯磺酰腙作为重氮化合物的前体可以很好地 解决上述问题, 参与一系列的卡宾偶联反应 ${ }^{[7]}$. 中国科 学院福建物质结构研究所黄学良团队在前期的工作中, 发展了钯催化卡宾桥联的 $\mathrm{C}-\mathrm{H}$ 活化反应策略(Carbene Bridging $\mathrm{C}-\mathrm{H}$ Activation), 可应用于异香豆素 ${ }^{[8]}$ 和中环 内酯的合成 ${ }^{[9]}$. 最近, 他们进一步将这一反应模式应用 于二苯并 $[b, e]$ 氮杂草-6-酮衍生物的合成 ${ }^{[10]}$, 通过逆合 成分析将二苯并七元含氮杂环结构分解成两个简单的 片段. 在钯催化下, 使用邻卤苯甲醛和 2-胺基- $N$-对甲苯 磺酰腙作为原料, 经由形式上的 $[4+3]$ 环加成反应快速
构筑这类含氮七元杂环骨架分子, 为一系列生物活性分 子和药物分子提供了模块化的合成工具.

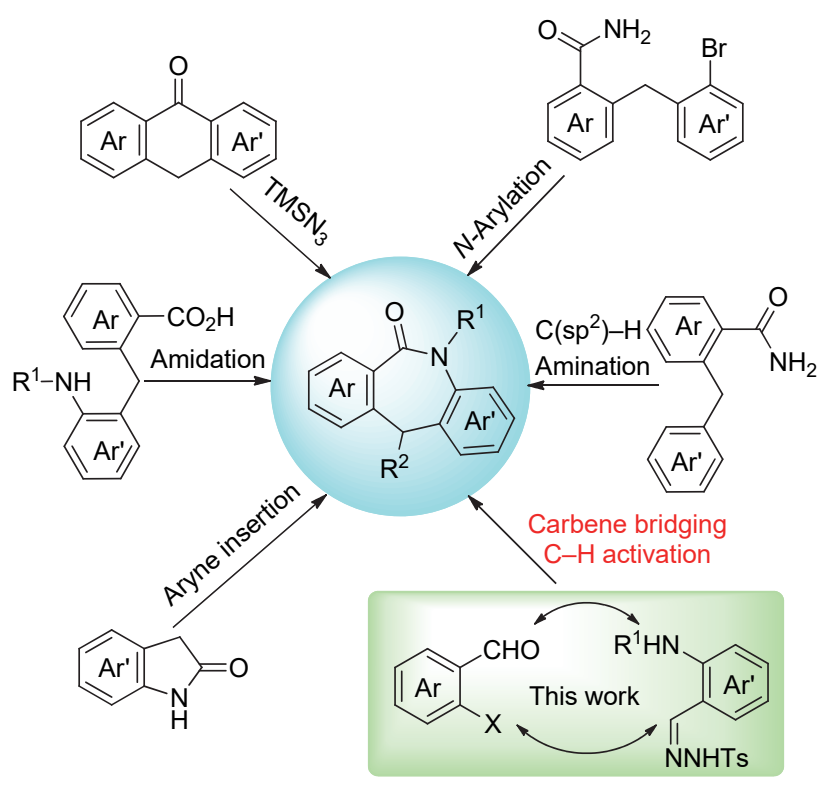

图式 1 构筑二苯并 $[b, e]$ 氮杂草-6-酮骨架分子

Scheme 1 Construction of dibenzo[b,e]azepin-6-one framework

该反应的原料简单易得，底物范围广(包括呋喃、噻 吩、吡啶和吲哚等杂环)，官能团兼容性好(包括羟基、 炔基和烯丙基等), 放大量实验收率也较好, 进一步可 以用于复杂分子的后期多样化衍生反应(如雌酮、扑热 息痛和 $L$-酪氨酸甲酯)(Scheme 2).

值得提出的是，该反应的产物进行一系列的衍生可 以构筑具有生物活性的含二苯并 $[b, e]$ 氮杂草骨架的分 子库. 如二苯并 $[b, e]$ 氮杂草-6-酮经过氨基脱保护, 与 1 -

* Corresponding author. E-mail: xiayingscu@scu.edu.cn. Published online October 24, 2020. 
甲基哌嗪缩合可以合成抗精神病药(Perlapine, Fluperlapine 和 Tilozepine); 经过铜催化的茮位氧化, 硼氢化钠 还原和烷基化可以合成抗惊厥药(Etazepine); 经过氢化 铝锂还原和二氧化锰氧化脱水生成的 $11 H$-二苯并 $[b, e]$ 氮杂草能进一步衍生合成抗组胺药(Epinastine)和抗抑 郁药(Mianserin). 此外, 这一形式上 $[4+3]$ 环加成反应 作为关键步骤可以用来实现维生素 A 增效剂 HX640 的 合成. 从苯酚出发, 用 2-胺基- $N$-对甲苯磺酰腙作为关键 原料, 仅需 8 步以 $44 \%$ 的总收率得到 HX640 (Scheme 3).
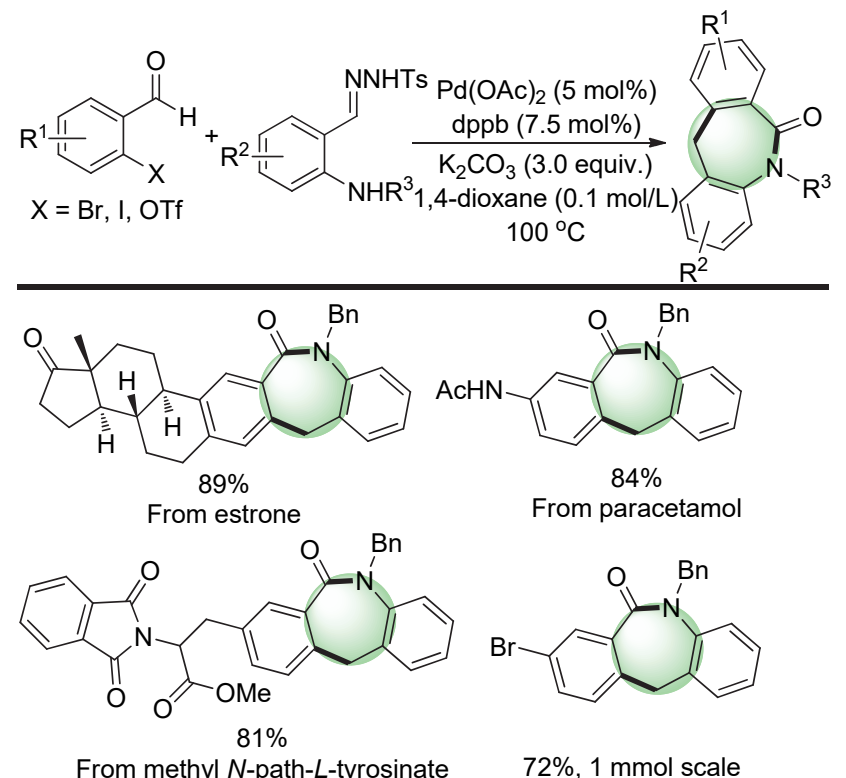

图式 $2[4+3]$ 环加成反应构筑二苯并 $[b, e]$ 氮杂草-6-酮骨架分 子

Scheme 2 [4+3] annulation to construct dibenzo[b,e]azepin-6ones

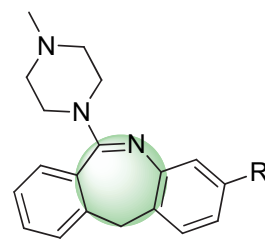

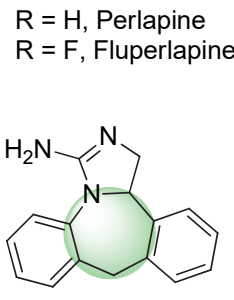

Epinastine

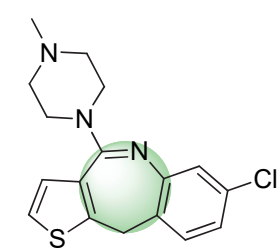

Tilozepine

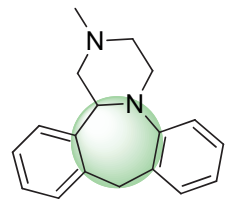

Mianserin

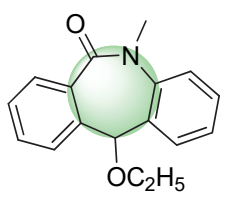

$\mathrm{HO}_{2} \mathrm{C}$ Etazepine

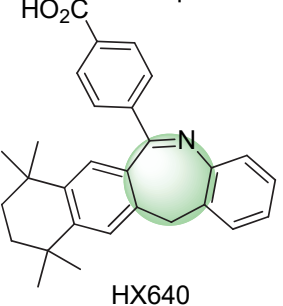

图式 3 产物衍生合成生物活性分子

Scheme 3 Derivatizations to construct bioactive molecules

氛代实验表明产物双苄位亚甲基上的两个氢原子 分别来自两个原料腙以及醛相连的氢, 从而支持反应经
历金属卡宾转移插入引发的 1,4-钯迁移过程 ${ }^{[11]}$ (Scheme 4). 零价钯首先和邻卤苯甲醛氧化加成生成二价芳基钯 物种 $\mathbf{I}$, 和原位生成的重氮化合物反应生成钯卡宾物种 II, 接着经过卡宾转移插入过程生成茮基钯物种 III, 随 后发生 1,4-钯迁移生成酰基钯物种 IV, 最后还原消除生 成内酰胺产物, 完成催化循环. 该反应以卡宾转移插入 生成的苄基钯物种为桥连，经由 1,4-钯迁移实现了远端 醛基碳氢键的活化. 在这一反应模式下，通过对 $N$-对甲 苯磺酰腙的 2 位引入不同的亲核基团, 有望实现各种含 有二苯并多元杂环结构的三环类化合物的合成.

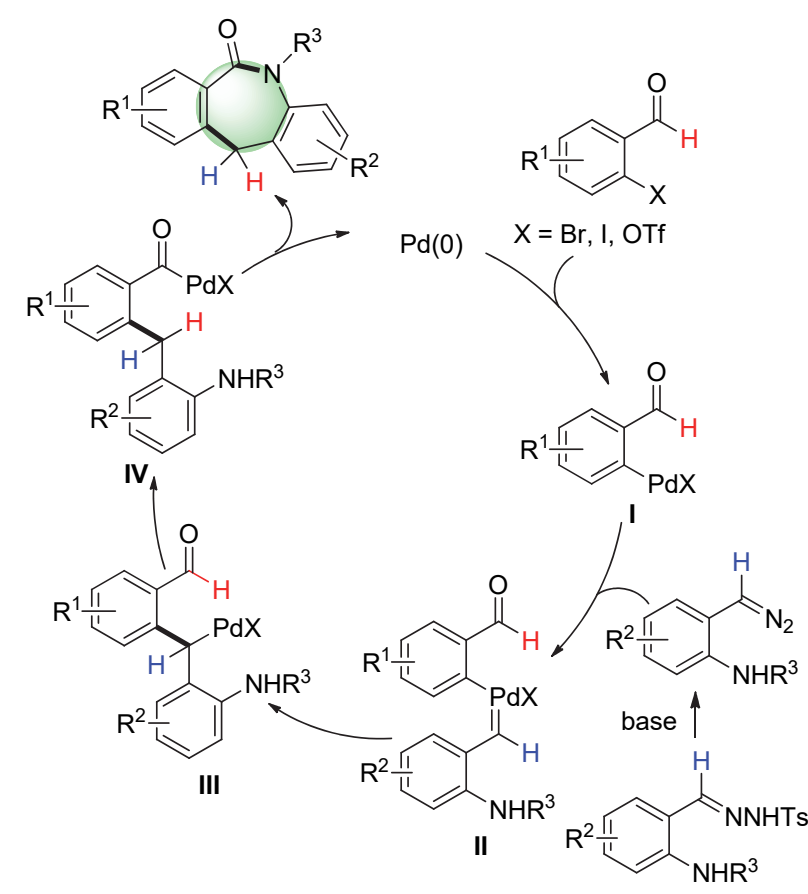

图式 4 可能的催化循环图

Scheme 4 Proposed catalytic cycle

\section{References}

[1] Wikstrçm, H. V.; Mensonides-Harsema, M. M.; Cremers, T. I. F. H.; Moltzen, E. K.; Arnt, J. J. Med. Chem. 2002, 45, 3280.

[2] Bunce, R. A.; Schammerhorn, J. E. J. Heterocycl. Chem. 2006, 43, 1031.

[3] Laha, J. K.; Shah, P. U.; Jethava, K. P. Chem. Commun. 2013, 49, 7623.

[4] Zhu, C.; Liang, Y.; Hong, X.; Sun, H.; Sun, W. Y.; Houk, K. N.; Shi, Z. J. Am. Chem. Soc. 2015, 137, 7564.

[5] Qin, C.; Zhou, W.; Chen, F.; Ou, Y.; Jiao, N. Angew. Chem., Int. Ed. 2011, 50, 12595.

[6] Samineni, R.; Bandi, C. R.; Srihari, P.; Mehta, G. Org. Lett. 2016, 18,6184 .

[7] Xia, Y.; Qiu, D.; Wang, J. Chem. Rev. 2017, 117, 13810.

[8] Yu, Y.; Lu, Q.; Chen, G.; Li, C.; Huang, X. Angew. Chem., Int. Ed. 2018, 57, 319.

[9] Yu, Y.; Chakraborty, P.; Song, J.; Zhu, L.; Li, C.; Huang, X. Nat. Commun. 2020, 11, 461.

[10] Yu, Y.; Ma, L.; Xia, J.; Xin, L.; Zhu, L.; Huang, X. Angew. Chem., Int. Ed. 2020, 59, 18261.

[11] Rahim, A.; Feng, J.; Gu, Z. Chin. J. Chem. 2019, 37, 929. 